\title{
Polycythaemia Vera among Sudanese Patients with Special Emphasis on JAK2 Mutations
}

\author{
Ibrahim Khidir Ibrahim ${ }^{1,2 *}$, Rosline Hassan², Elshazli Widaa Ali', Awad Omer ${ }^{3}$
}

\begin{abstract}
Background: In recent years, a somatic point mutation in the Janus Kinase 2 (JAK2) gene (1849 G $\rightarrow \mathrm{T}$, V617F) has been reported to occur in over $90 \%$ of patients with polycythemia vera (PV). Another JAK2 mutation in exon 12 had been described and shown capable of activating erythropoietin signaling pathways. Objective: In this study, we aimed to determine the frequency of Jak2 mutations (JAK2V617F and JAK2 exon 12) as well as their relationships with hematological parameters in Sudanese patients with myeloproliferative disorders (MPD). A comparison with findings of published studies from other geographic regions was included. Materials and Methods: From each of a total of 83 polycythaemia patients, six milliliters $(\mathrm{ml})$ of venous blood were collected and processed for molecular analysis and measurement of serum erythropoietin level by enzyme-linked immunoassay (ELISA). The JAK2 V617F mutation was determined using an allele-specific competitive blocker (ACB) -PCR assay and High Resolution Melting (HRM) analysis was applied for the JAK2 exon 12 mutation. Results: According to patients' history and the results for EPO levels, nine (10.7\%) out of 83 patients were found to have secondary polycythaemia and $74(89.3 \%) \mathrm{PV}$. The overall frequency of the 2 JAK2 mutations was $94.6 \%$ in our Sudanese PV patients, JAK2V617F being found in 91\% and JAK2 exon 12 mutations in 8.1\%.Conclusion: In summary JAK2 V617F and JAK2 exon 12 mutations are very common in Sudanese PC cases.
\end{abstract}

Keywords: Polycythaemia vera- erythropoietin- JAK2 mutation

Asian Pac J Cancer Prev, 20 (1), 41-44

\section{Introduction}

True polycythaemia refers to an absolute increase in total body red cell volume (or mass), which usually manifests itself as a raised haemoglobin $(\mathrm{Hb})$ concentration and packed cell volume (PCV). A raised $\mathrm{Hb}$ (or PCV) can also be secondary to a reduction in plasma volume, without an increase in total red cell volume; this is known as apparent (or relative) polycythaemia. True polycythaemia is further subdivided into primary polycythaemia (polycythaemia vera), a clonal haematological disorder, and secondary Polycythaemia (also known as erythrocytosis); which results from an increased erythropoietin hormone, either in the presence or in the absence of hypoxia (Hoffbrand et al., 2016).

The Janus kinases are a family of large cytoplasmic tyrosine kinases with molecular weights in the range of 120-140 kDa (1130-1142 aa). In mammals, there are four members of the Jak family: Jak1, Jak2, Jak3, and Tyk2.15 From C-terminal to N-terminal, Jaks consist of seven conserved domains, termed Jak homology (JH) domains 1-7. JH1 and $\mathrm{JH} 2$ domains exert the most important functions of Jaks. JH1 acts as a kinase domain, containing the ATP-binding region and the activation loop. The $\mathrm{JH} 2$ domain is the pseudokinase domain, which is highly homologous to tyrosine kinase domain, but lacks the catalytic activity due to the absence of necessary residues. The pseudokinase domain is believed to have autoinhibitory function and regulate both basal activity of the Jak kinases and cytokine-induced activation of the catalytic function (Saharinen et al., 2000; Saharinen et al., 2003).

Normally, the JAK/STAT pathway is tightly controlled to ensure normal blood cell production and function, but disruptions in the pathway can cause disease states. In 2005, several independent groups used different experimental approaches to identify a recurrent mutation in the JAK2 tyrosine kinase in most patients with PV, Essential thrombocythaemia or Primary myelofibrosis (Levine, 2005; Kralovics, 2003). The mutation is a G-T substitution at nucleotide 1849 in codon 617 of the JAK2 gene which results in substitution amino acid valine-to-phenylalanine that confers constitutive tyrosine kinase activity to the mutant gene.

As Scott et al., (2007) described different mutations in exon 12 of JAK2 among V617F-negative patients

${ }^{1}$ Department of Haematology, Faculty of Medical Laboratory Sciences, Al-Neelain University, ${ }^{3}$ Royal Care International Hospital, Khartoum, Sudan, ${ }^{2}$ Department of Haematology, School of Medical Sciences, University Sains Malaysia, Health Campus, 16150 Kubang Kerian, Kelantan Malaysia.*For Correspondence: ibrahimkh82@gmail.com 
with polycythaemia vera or idiopathic erythrocytosis in 2007. It has been approved that JAK2 exon 12 mutations can activate signaling pathways of erythropoietin. These findings have been confirmed by many studies conducted among different ethnic group from many countries, (Ma et al., 2009; Pietra et al., 2007) but there are no reports from Sudan about the frequency of the JAK2 mutations in patients with PV. In the present study, we determined the frequency of JAK2V617F mutation and JAK2 exon 12 mutations in patients with PV in Sudan.

\section{Materials and Methods}

\section{Patients and Samples}

A total of 83 Sudanese patients attended Khartoum teaching hospital and Military hospital with HCT greater than $52 \%$ for males and $48 \%$ for females were enrolled in this study; $74(92 \%)$ of them were males and nine (8\%) were females; age range between 23-80 years. Six milliliter $(\mathrm{ml})$ of venous blood were collected from each patient, $3.0 \mathrm{ml}$ in ethylene diamine tretraacetic acid (E.D.T.A) container for complete blood count and molecular analysis and $3.0 \mathrm{ml}$ in plain container for measurement of serum erythropoietin level.

\section{Measurement of serum erythropoietin level}

Serum erythropoietin level was measured using enzyme -linked immune assay (ELISA) technique for each patient using "WEKA HUMAN EPO ELISA KIT, USA" and according to manufactures instructions.

\section{DNA Extraction}

Genomic DNA was extracted from an EDTA blood sample using a kit (GFI-1 BLOOD DNA EXTRACTION KIT, Malaysia) according to the manufacturer's instructions.

\section{JAK2V617F Mutation Analysis}

The JAK2 V617F mutation was detected using Allele-specific competitive blocker (ACB)-PCR assay (Tan et al., 2007). The cycling conditions were $95^{\circ} \mathrm{C}(5$ minutes) and 45 cycles of $94^{\circ} \mathrm{C}(30$ seconds $), 64^{\circ} \mathrm{C}(30$ seconds), $72^{\circ} \mathrm{C}$ (30 seconds), and a final $72^{\circ} \mathrm{C}$ extension for 10 minutes. Five $\mu$ l of the PCR product (ready to load) was electrophoresed on 3\% Agarose gel, and was stained with ethedium bromide. The presence of the 139-base-pair (bp) band indicated that the sample was positive for the JAK2 V617F mutation (mutated type) whereas the absence of the band indicates that sample was negative for the mutation (wild type).

\section{Detection of JAK2 exon 12 mutation}

JAK2 exon 12 mutations were detected by high resolution melting (HRM) analysis technique Using HRM type In kit, JAK2 exon 12 specific primers (Rapado et al., 2009) were used and amplified according to the manufacturer's instructions using Rotor gene-Q real time PCR. Fluorescence was detected continuously. Data were analyzed using the Rotor-Gene Q software (QIAGEN,
Hilden, Germany). Samples with aberrant melting curves were recorded as HRM mutation positive.

\section{Statistical analysis}

The statistical analysis of laboratory results and patients' data were done using the SPSS version 21. Frequency of JAK2V617Fmutation determined by descriptive statistical analysis. Independent sample T-test was used to compare the peripheral $\mathrm{RBC}, \mathrm{WBC}$, and platelet counts at diagnosis between patients with a JAK2 mutations and those without the JAK2 mutations.

\section{Ethical consideration}

Ethical approval was obtained from the ethical committee of Al-Neelain University and informed consent Informed consent was taken from all the patients prior to their inclusion in the study.

\section{Results}

A total of 83 patients attending the military hospital, and Khartoum teaching hospital, presented with absolute erythrocytosis were enrolled in this study. Their ages ranged between 23-80 years (mean \pm SD: $39 \pm 1.5$ ). According to patients' history and the results of EPO level, nine (12.1. \%) out of 83 patients were found to have secondary polycythaemia (normal or increased erythropoietin) patient was found to have secondary polycythaemia (SP) (increased erythropoietin level), and $74(87.9 \%)$ of patients had polycythaemia Vera (PV) (decreased serum erythropoietin level).

The frequency of investigated JAK2 V617F mutation was detected in $67(91.0 \%)$ out of 74 (eleven of them were females and fifty sex were males) studied Sudanese polycythaemia Vera patients, while it was not detected in seven $(9.5 \%)$ of them and all of them were males.

The overall mean age of the studied group was 38 . Moreover the mean age at presentation was insignificantly lower in JAK2V617F-positive patients (mean 42 STD+ 14.3) than those JAK2V617F-negative (mean 43 STD +13.6) $\mathrm{p}<0.75$ ). The Most frequent decade was fourth decade.

Mean of HCT was found slightly higher (51.2 \pm 2.5$)$ in patients with JAK2V617F mutation than those negative for the mutation (51.1 \pm 3.5$)$. Mean of TWBcs and platelets count $(7.39 \pm 3.1$ and $352 \pm 180$ respectively) was found higher in patients with JAK2V617F mutation compared with those negative for the mutation (5.4. \pm 0.93 and $260 \pm, 85$ respectively); but this difference between the two groups in HCT, TWBcs and platelets was statistically insignificant (P.Value: 0.92, 0.184, and 0.09 respectively).

Mean of HCT was found slightly higher (52.8 \pm 3.0$)$ (Mean $\pm \mathrm{SD}$ ) in patients with JAK2 exon 12 mutation than those negative for the mutation (51.0 \pm 2.5$)$. Mean of TWBes and platelets count (6.7 \pm 3.2 and $279 \pm 156$ respectively) was found lower in patients with JAK2 exon 12 mutation compared with those negative for the mutation (7.2 \pm 3.0 and $349 \pm 176$ respectively). 


\section{Discussion}

Distinction of PV from secondary polycythemia has remained a complicated task during the evaluation of erythrocytosis. Due to differences in clinical profile, disease evolution, prognostic implications and therapeutic options, the significance of differentiating PV from secondary polycythemia can't be ignored..

Discovery of the JAK2 V617F mutation in myeloproliferative disorders in 2005 has enabled us to understand the molecular and cellular basis of these disorders. There is considerable variation in the reports of JAK2V617F frequency in MPD, with incidence rates of $23-57 \%$ in essential thrombocythemia, 35-57\% in idiopathic myelofibrosis and 65-97\% in polycythemia vera (PV). The revised WHO criteria for diagnosis of $\mathrm{PV}$ in 2008 include JAK2V617F mutation as one of the major diagnostic criteria for of PV.

The present study revealed that the mean age of incidence in Sudanese patients with polycythaemia Vera was in the fourth decade and this finding disagrees with findings from a study done by Sazawal et al., (2010) in India which reported that mean age of incidence of polycythaemia Vera is in the fifth decade. Also the present study disagrees with study done in India (Karakus, 2012) which reported that Median age at diagnosis was 60 years. In agreement with the fact that polycythaemia Vera was not reported in patients under 20 years old, none of our patients was aged less than 20 years.

The frequency of JAK2V617F mutation among Sudanese patients with PV in this study was found to be $91 \%$. This finding was consistent with that reported in Turkish (89.3\%), Pakistanian (93.5\%) and Saudi Arabian patients (91\%) (Gari et al., 2012).

A study done by Ayad and Nafea, (2011) in Egypt reported that, frequency of the JAK2V617F mutation in polycythemia vera was $81.4 \%$. Another study done by Yeh $(, 2010)$, reported a frequency of $77 \%$ in Taiwan patients. These finding are lower than those among Sudanese patients.

The frequency of JAK2 exon 12 mutations among Sudanese patients with PV in this study was found to be $8.3 \%$, which is consistent with results reported by $\mathrm{Li}$ et al., (2008) who conducted study in China and reported frequency of an $8.5 \%$. The results of the study also disagree with findings of the study done by Santos et al., (2013) who reported that the frequency of JAK2 exon 12 mutations among patients with PV in Brazil was $2 \%$.

It is obvious that the Results of many studies concerning with the frequency of JAK2 exon 12 mutations among polycythaemia Vera patients in different populations show conflicting results. This conflict in reported rates is likely to be due to differences in diagnostic precision, assay sensitivity and ethnic variations.

A possible reason for discrepancies in the observed mutation frequency in PV by different research groups includes differences in assay sensitivity, diagnostic inaccuracy and ethnic variation.

In the present study, HCT, leucocyte count and platelets count were compared in polycythaemia Vera patients' positive for JAK2V617F mutation and those negative for the mutation; Leucocyte count and platelet count were higher in those with the mutation than those without the mutation but the difference was not statistically significant, while HCT was similar in both groups. These findings are consistent with the results reported by Gari et al., (2012) that total leucocyte count and platelets count were higher in patients with the JAK2-V617F mutation but we are inconsistent with them in their findings that HCT was higher in mutated group.

In the present study, HCT, leucocyte and platelets counts were compared in studied polycythaemia Vera patients positive for JAK 2 exon 12 mutation and those without the mutation HCT was higher among patients with the mutation than those negative for mutation while Leucocyte count and platelet count were lower in those with the mutation than those without the mutation but the differences were not statistically significant in both cases. These findings are consistent with results published by Scott et al., (2007) who reported that British patients with JAK2 exon 12 mutations presented with isolated erythrocytosis.

In this study we found that the overall frequency of JAK2 mutations in Sudanese patients with polycythaemia Vera was $94.6 \%$, and there is coexistence of JAK2V617F and JAK2 exon 12 mutations in three patients (4.05\%). This finding is consistent with results reported by $\mathrm{Li}$ et al., (2008) who conducted a study in Washington and reported that, the overall frequency of JAK2 mutations in studied patients was $93 \%$ and there was $1.4 \%$ patient with coexistent mutation.

Seventy and half percent of patients with JAK2V617F mutation presented with thrombocytosis and $12.1 \%$ presented with leukocytosis in comparison all patients with JAK2 exon 12 mutations presented with normal leucocyte count and platelets count. All patients negative for JAK2V617F mutation presented with normal leucocyte and platelets count at diagnosis.

In Conclusion, JAK2 mutations is detected in $94.5 \%$ of the studied Sudanese polycythaemia Vera patients. The data indicated an exemplified the importance of JAK2V617F mutation and JAK2 exon 12 mutation analysis in a clinical laboratory for the diagnosis of PV.

\section{References}

Ayad M, Nafea D (2011). Acquired mutation of the tyrosine kinase JAK2V617F in Egyptian patients with myeloid disorders. Genet Test Mol Biomarkers, 15, 17-21.

Gari M, Al-Sayes F, Ahmed F, et al (2012). JAK2 mutations in chronic myeloproliferative neoplasm; towards the application of personalized treatments for Saudi patients. Open J Blood Dis, 2, 17-21.

Hoffbrand A, Higg, D, Keeling D, Mehta A (2016). Postgraduate haematology. Chichester, West Sussex: John Wiley and Sons, Inc.

Karakus S (2012). JAK 2V617F mutation: frequency and relation to clinical and laboratory features of BCR-ABL negative myeloproliferative diseases. Int J Hematol Oncol, 22, 77-84.

Kralovics R (2003). Comparison of molecular markers in a cohort of patients with chronic myeloproliferative disorders. Blood, 102, 1869-71.

Levine R (2005). The JAK2V617F activating mutation occurs 
in chronic myelomonocytic leukemia and acute myeloid leukemia, but not in acute lymphoblastic leukemia or chronic lymphocytic leukemia. Blood, 106, 3377-9.

Li S, Kralovics R, De Libero G, et al (2008). Clonal heterogeneity in polycythemia vera patients with JAK2 exon12 and JAK2-V617F mutations. Blood, 111, 3863-6.

Ma W, Kantarjian H, Zhang X, et al (2009). Mutation profile of JAK2 transcripts in patients with chronic myeloproliferative neoplasias. J Mol Diagn, 11, 49-53.

Rapado I, Grande S, Albizua E, et al (2009). High resolution melting analysis for JAK2 exon 14 and exon 12 mutations. J Mol Diagn, 11, 155-61.

Saharinen P, Takaluoma K, Silvennoinen O (2000). Regulation of the Jak 2 tyrosine kinase by its pseudokinase domain. $\mathrm{Mol}$ Cell Biol, 20, 3387-95.

Saharinen P, Vihinen M, Silvennoinen O (2003). Autoinhibition of Jak2 tyrosine kinase is dependent on specific regions in its pseudokinase domain. Mol Biol Cell, 14, 1448-59.

Santos MTD, Mitne-Neto M, Miyashiro K, et al (2013). Molecular genetic tests forJAK2V617F, Exon12 JAK2 and MPLW515K/L are highly informative in the evaluation of patients suspected to have BCR-ABL1-negative myeloproliferative neoplasms. J Clin Pathol, 67, 176-8.

Sazawal S, Bajaj J, Chikkara S, et al (2010). Prevalence of JAK2 V617F mutation in Indian patients with chronic myeloproliferative disorders. Indian J Med Res, 132, 423-7.

Scott LM, Tong W, Levine RL, et al (2007). JAK2 exon 12 mutations in polycythemia vera and idiopathic erythrocytosis. N Engl J Med, 356, 459-68.

Tan A, Westerman D, Dobrovic A (2007). A simple, rapid, and sensitive method for the detection of the JAK2V617F mutation. Am J Clin Pathol, 127, 977-81.

Yeh Y, Chen Y, Cheng H, et al (2010). High percentage of JAK2 exon 12 mutation in Asian patients with polycythemia vera. Am J Clin Pathol, 134, 266-70.

This work is licensed under a Creative Commons AttributionNon Commercial 4.0 International License. 